\title{
Simulation Approach to Life-Data Queue Event Modeling
}

\author{
Johnson O.V* and Ganiyu M. \\ Department of Computer Science, The Federal Polytechnic, Ile-Oluji, Nigeria \\ Simulation process provides a platform to model the real-life scenario from an experimental viewpoint. Simulation \\ plays a key role in providing output that could be used to model the real-life. Queue patterns are studies from two \\ perspective: (1) the stochastic method and (2) simulation technique. This paper spurs on discrete event simulation \\ (DES) technique to investigate the assertions already made in queue model research works. Life-Data already collected \\ from Johnson et al (2018) was used and simulation carried out using simmer package in R language. Findings validate
}

\begin{abstract} the result of assertions made some research literatures.

Keywords: Simulation, Queue, Discrete, Simmer, assertions

DOI: $10.7176 / \mathrm{CTI} / 8-04$
\end{abstract}

\section{Introduction}

Queue, is a general and practicable event of everyday life visible in human activities. They exist in day to day human activities where line is to be whenever demand for a service is required and have to wait in that their number exceeds the number of servers available; or the facility does not work efficiently or takes more than the time prescribed to service a customer. Some customers wait when the total number of customers requiring service exceeds the number of service facilities, some service facilities stand idle when the total number of service facilities exceeds the number of customers requiring service. Taha, (2003) defined as simply as people or event waiting in line, while Hiray, (2008) puts it in similar way as a waiting line by two important elements: the population source of customer from which they can draw and the service system. The population of customer could be finite or infinite.

A major human activity in our business environment today is customers seen queueing at Point-of-Sales (POS) point to perform one or two services. One of such points is the Automatic Teller Machine (ATM) at banking hall or designated locations. Meeting the need of customer requires, therefore, a seamless hitch-free availability of service system. (Johnson O.V et al, 2018). Customers might be dissatisfied with service rendered whenever much delay is being experienced at service point. Customers have tendency to wait in line as much as the server is performing and the line is moving. Therefore, strict attention and adherence must be paid to ensuring optimum service delivery as banking system intend to make profit and still remain in business.

Queue studying and modeling are answers to providing decision maker of banking operation as to best practices that could be implore to lessen the dilemma of incessant service failure of Atm to minimize such. Too much waiting is costly. An aspect of computational mathematics: queueing theory is therefore, adopted in solving waiting line problem and analyzing congestions and delays of waiting in line problem (Investopedia, 2014). Queueing theory examines every component of waiting in line to be served, including the arrival process, service process, number of servers, number of system places and the number of "customers" (which might be people, data packets, cars, etc.).

The experimental design and case study were carried out at Rufus Giwa Polytechnic, Owo, Ondo State in south-west, Nigeria. The massive increase in infrastructural development ranging from opening of new sites for construction, lecture halls and offices, diverse renovations to establishment of new faculties to mention but a few brought about population of 14,000+ estimate. A huge demand was placed on facilities especially the ATM. This necessitated that proper planning on ATM deployment and utilization must be forthwith put in place.

Drawing from the research carried out by Johnson et al (2018) on queueing planning model using stochastic solution method of queue theory from the above case study, results show that available service points (ATM) considered are being over stressed. Longer than enough queues were visible in the system with its poor service performance. There were possibility of customers reneging and other conflicts with its intending vices. Meanwhile one cannot come into a final conclusion about the need to deployment more ATMs as solution to the challenge investigated by the aforementioned authors.

The use of simulation as a prediction process to real life modeling is presented in this paper to investigate the data already generated, compare the result and a provide with a probable solution to the challenge. 


\section{Aim and Objectives of the Study}

The major focus of this research work was to investigate the outcome of the work carried out by Johnson et al (2018) on ATM performance and utilization on the need to deploy more service point and the queue model deployment strategy that should be adopted by using discrete-event simulation technique rather than the stochastic solution method.

\section{Related Work}

Johnson et al (2018) presented a queue model using stochastic solution method on a real-life queue data collected from at ATM service point. The research was contrary to other works that placed assumption on arrival and service pattern without real-life data to proof. Although investigated result shows that ATM resources were over utilization, reneging and conflict are common place. They proposed the deployment of four to five additional ATM points.

A research work of Vasumathi. et al (2010) considered a simulation technique on 3 ATM services of 3 different Banks at Vellore Institute of Technology, Chennai. Data were sourced from these banks using observation method in a period of 2 months. Their result shows a low service delivery in some area of the campus and suggested a new installation despite its cost.

Bakari et al (2005) did a research work on queueing process and its application to customer service delivery (a case study of Fidelity Bank Plc, Maiduguri). Observation method was primarily their method of data collection over a period of 10 working days. The study reveals that the traffic intensity $(\rho)$ is 0.96 , otherwise known as the utilization factor is less the one (i.e. $\rho<1$ ). It was concluded that the system operates under steady-state condition. Thus, the value of the traffic intensity, which is the probability that the system is busy, implies that $96 \%$ of the time period considered during data collection the system was busy as against $4 \%$ idle time. This indicates high utilization of the system.

\section{Simulation and Queue System}

In most cases simulation tends to show how a system would look like in a real world. It is also a way of comparing result with the stochastic solution if practically available in order to justify the true nature of the stochastic solution. A discrete-event simulation (DES) is a process of modeling the operation and dynamism of a system as a discrete sequence of events in time or the behaviour of a complex system as an ordered sequence of a well-defined events (Wikipedia, retrieved Aug, 2017 and Prateek, 2015). A definition was also provided by Shannon (1975), stating simulation "as a process of designing a model of a real system and conducting experiments with this model for the purpose either of understanding the behaviour of the system or of evaluating various strategies (within the limits imposed by a criterion or a set of criteria) for the operation of the system." The process is that an event occurs in a set instant of time marking a change of state in the system. No change in the system is assumed to occur between consecutive events; meaning that the process can directly jump from one event to the next in the defined timing. This is contrast to continuous simulation in which the simulation continuously tracks the system dynamics over time, stochastic, deterministic, statics versus dynamic as common taxonomy models of simulation. Some applications in the category of DES include Queue system, Investment prediction, Network analysis and Hospital performance analysis (Babulak, 2008).

We present DES terminology below in order to understand the concept and its design:

i. Arrival: An arrival can be described as a process, an active entity which has number of activities associated to it and, in general, a limited lifetime in the system. These activities conform to a trajectory specification. It is created by a generator.

ii. Trajectory: When a generator creates an arrival, it couples the arrival to a given trajectory. A trajectory is defined as an interlinkage of activities which together forms the arrivals' lifetime in the system. Once an arrival is coupled to the trajectory, it will (in general) start processing the activities in the trajectory in the specified order and, eventually, leave the system.

iii. Activity: Different kind of activities exist that allow arrivals to interact with resources, perform custom tasks while spending time in the system, move back and forth through the trajectory dynamically. The set of variables of such activities consist of seize, release, timeout, set_attribute, rollback and branch.

iv. Generator: A generator is a process which create new arrivals with a given interarrival time pattern and inserting them into the simulation model.

v. Resource: A resource is in essence a passive entity. It comprises two parts: 
1) Server: it represents the resource itself. It has a specified capacity and can be seized and released by an arrival.

2) Queue: When an arrival tries to seize a resource (tries to access the server) and it is busy, this arrival is appended to the waiting line, if there is a room for it. If not, the arrival is rejected and immediately leaves the system.

vi. Manager An active entity, i.e., a process, that has the ability to adjust properties of a resource (capacity and queue size) at run-time.

There are couples of software and programming languages as tools available to perform simulation experiment for DES such as SimPy, SimJulia, Java etc. Meanwhile we implemented simmer package in R for this project. The simmer package brings discrete-event simulation to R. As a generic design, it is yet powerful process-oriented framework. The architecture encloses aa robust and fast simulation core written in C++ with automatic monitoring capabilities. It provides a rich and flexible R API that revolves around the concept of trajectory, a common path in the simulation model for entities of the same type. Figure 1 shows the UML diagram of the core architecture of simmer package in DES.

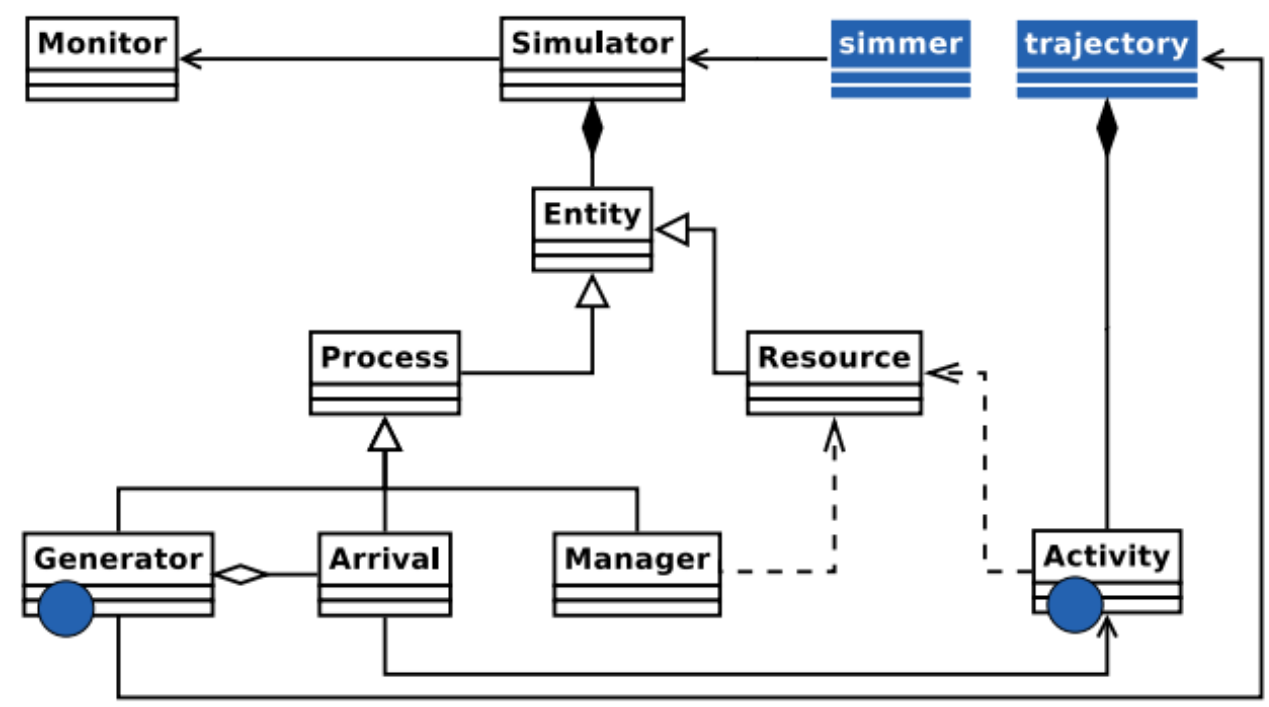

Figure 1: UML diagram showing the core architecture of simmer to discrete simulation event modeling (Adapted from: Ucar, Smeets and Azcorra (2017), simmer: Discrete-Event Simulation for R, arXiv:1705.09746v2 [stat.CO] 5 Dec 2017). 


\section{Computer Simulation of the Queue Model}

Figure 2 and 3 provide a flowchart of simulation procedures implemented in the paper. The arrival pattern is set within the process and simulation result could be generated as shown.

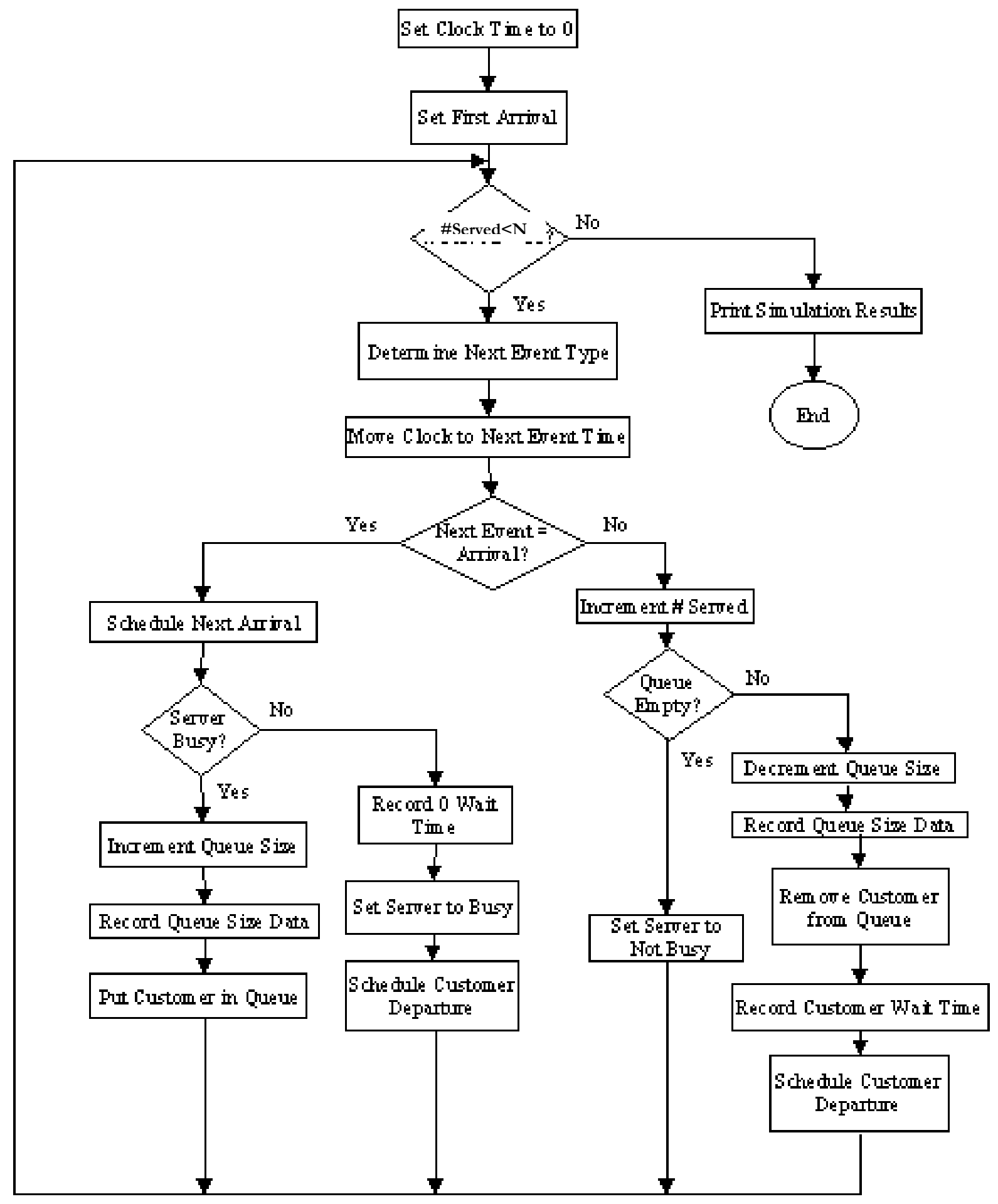

Figure 1.1 showing the queue model of the ATM with an improved n-customers adapted from Brian J. Huffman (2014) "An Object-Oriented Version of SIMLIB (a Simple Simulation Package)", School of Business University of Wisconsin - River $35^{\text {alls, }}$, retrieved $31^{\text {st }}$, Oct 2014 


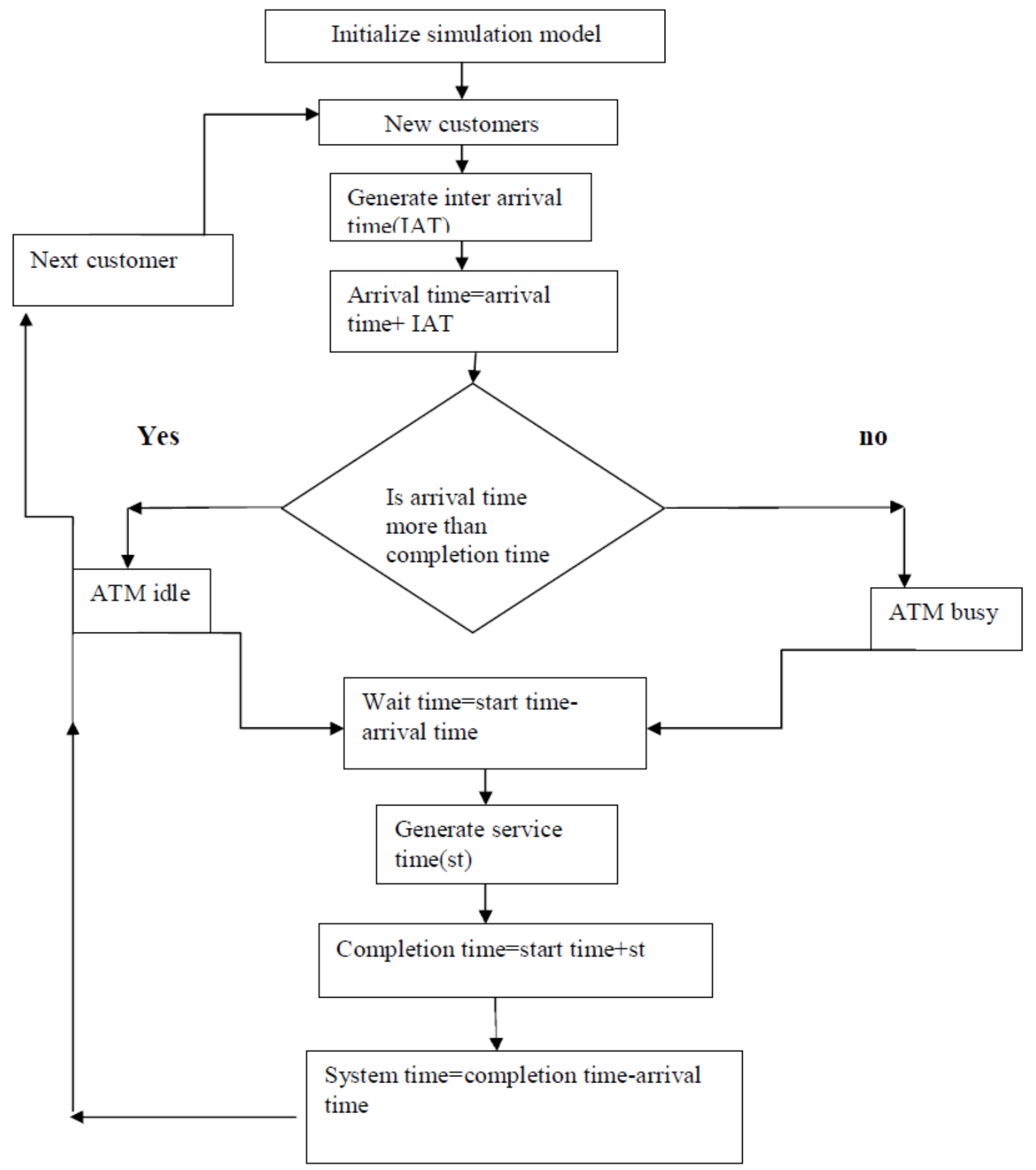

Figure 1.2 showing the simulation of ATM waiting line adapted from Vasumathi.A and Dhanavanthan P (2010)," Application of Simulation Technique in Queuing Model for ATM Facility", International Journal of Applied Engineering Research, Dindigul, Volume 1, no 3, 2010.

\section{Materials and Methods}

This paper adopted the data collected from Johnson et al (2018) work to investigate the outcome of their work using DES technique and drawing statistical inference on the assumptions and solutions provided in their work. The data set consist over 2000 queue event record. The summary of the data is provided in Table 1.0. A generalized M/M/c/ $/ \infty / F I F O$ model was adopted, where the first $\mathrm{M}$ denotes the inter-arrival time, the second $\mathrm{M}$ denotes the service time with $\mathrm{c}$ 
channels and First-In-First-Out discipline to analyze the data collected to derive the queue models and its parameters. Simmer package was used with program written in $R$ language. The basic DES algorithm is as given in algorithm 1.0 below.

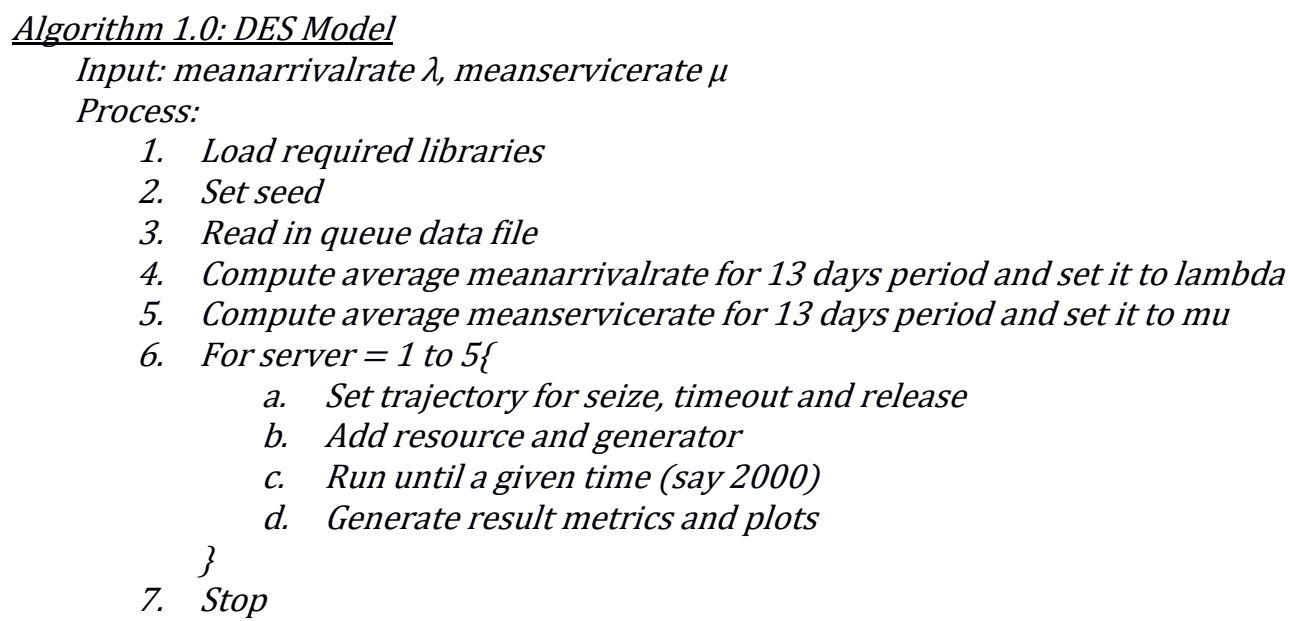

\section{Basic Queue Model Assumptions}

Model as an idealized representation of the real-life situation; in order to keep the model as simple as possible however, some assumptions need to be made (Hira and Gupta, 2004). The following assumptions is made on the System

1) Poisson arrival (Random arrivals).

2) Inter-arrival time \& Service time follow exponential distribution.

3) Multiple channel queue.

4) There is an infinite population from which customers originate.

5) The queue discipline is First-In- First-Out (FIFO).

6) The waiting area for customers is adequate.

\section{$7.1 \mathrm{M} / \mathrm{M} / \mathrm{c} / \infty /$ FIFO model}

Establishing a foundation for this work, M/M/c/ $\infty / F I F O$ model is analyzed with exponential interarrival times with mean $1 / \lambda$, exponential service times with mean $1 / \mu$ and a single serve. Customers are served in order of arrival. We require the utilization rate from

1) Customers in the sojourn time

2) Customers in the system

3) Service time in the sojourn time

4) Service time in the system

5) Waiting time in the sojourn time

6) Waiting time in the system

Thus

$$
\frac{\text { Average arrival time }}{\text { Average Service time }}=\frac{\lambda}{\mu}
$$

as

$$
\rho=\frac{\lambda}{c \mu}<1 \quad \rho=\left\{\begin{array}{r}
\text { not busys } \rho<1 \\
\text { busy. } \rho>1
\end{array}\right.
$$




\section{Simulation Results and Discussion}

Figure 4(a-e), 5(a-e) and 6(a-e) are presented below as graphical results of simulation process considering the capacity (Server) setup from 1 to 5 :
a. The average numbers of customers in the system

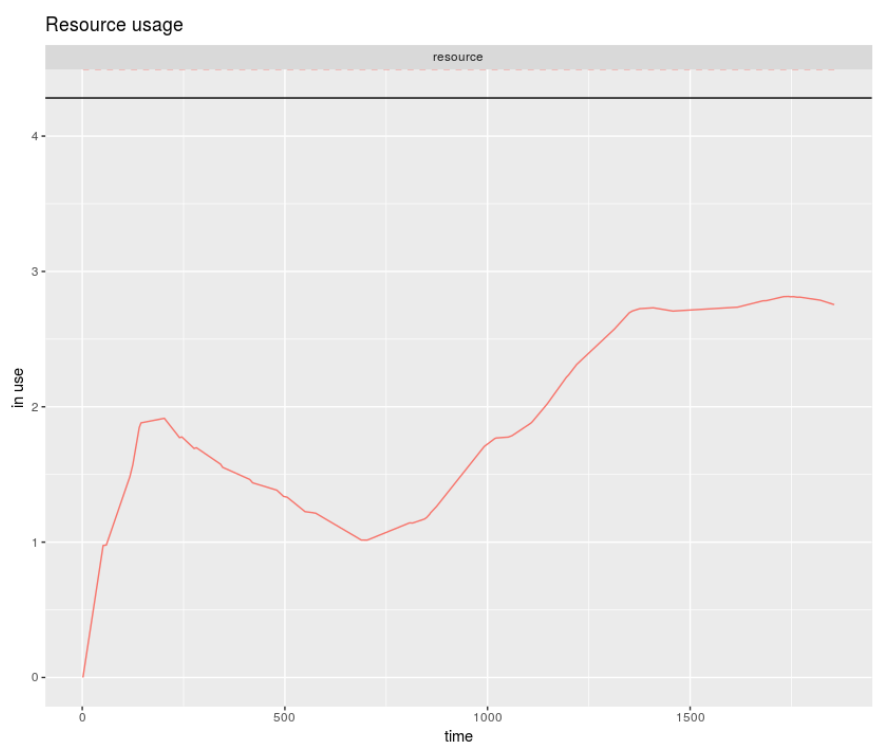

(a)

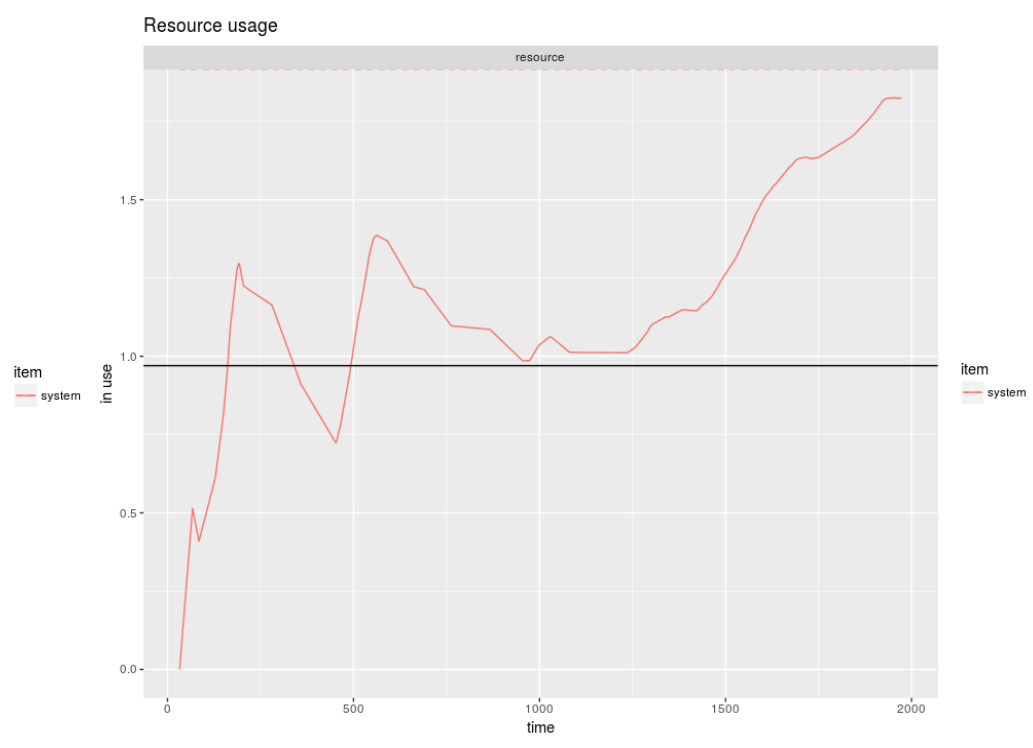

(b)

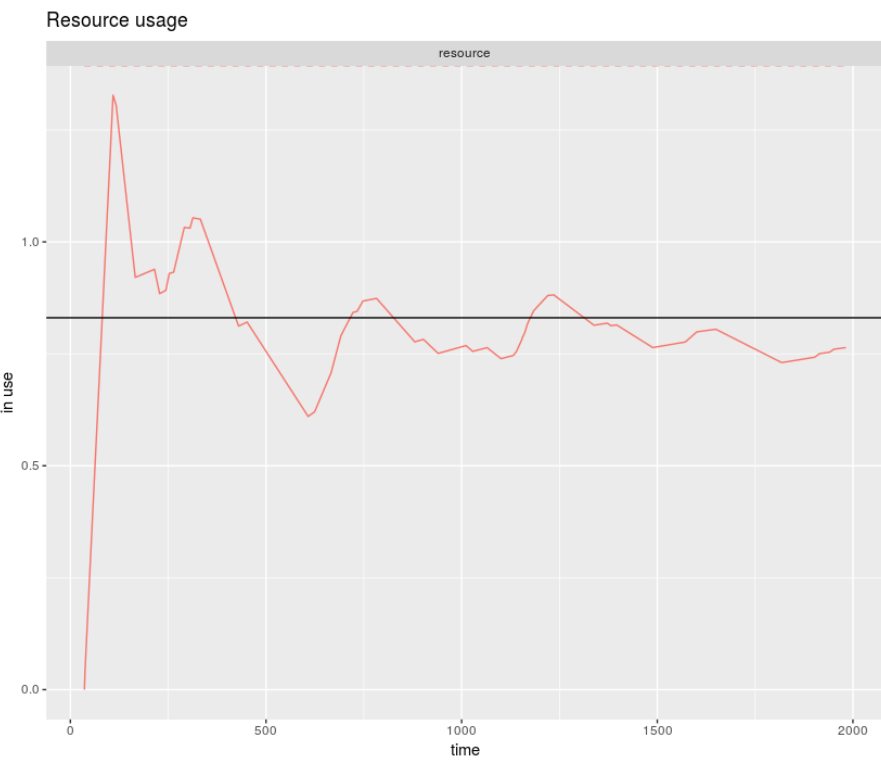

(c)

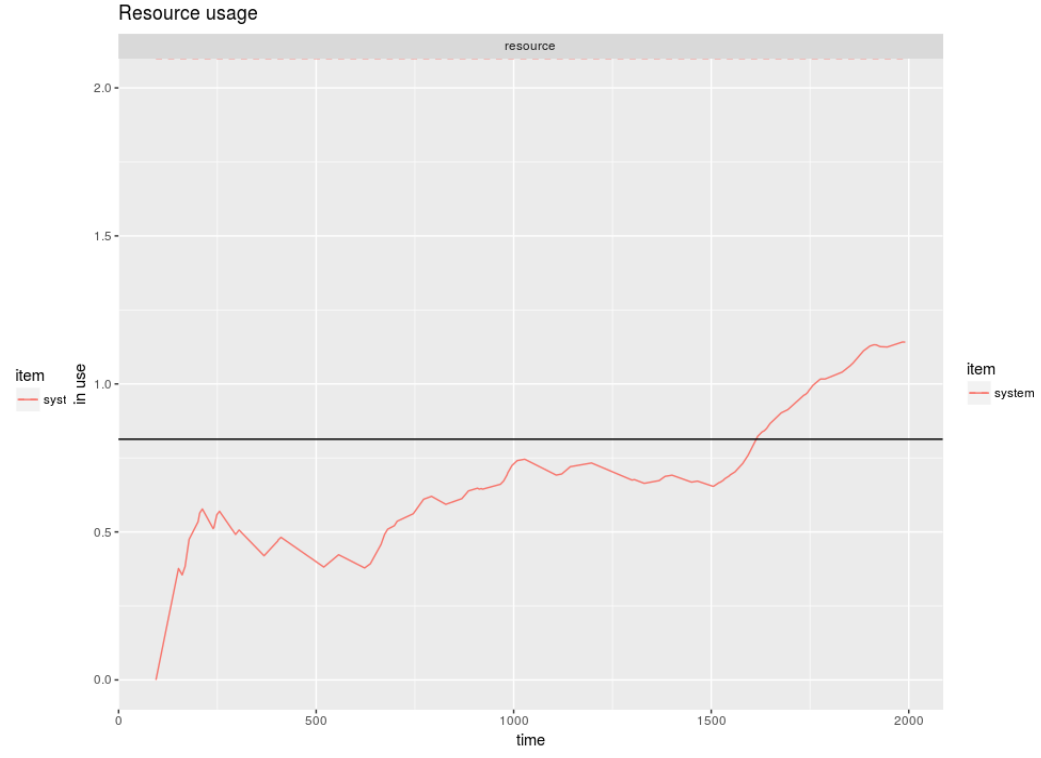

(d) 


ISE

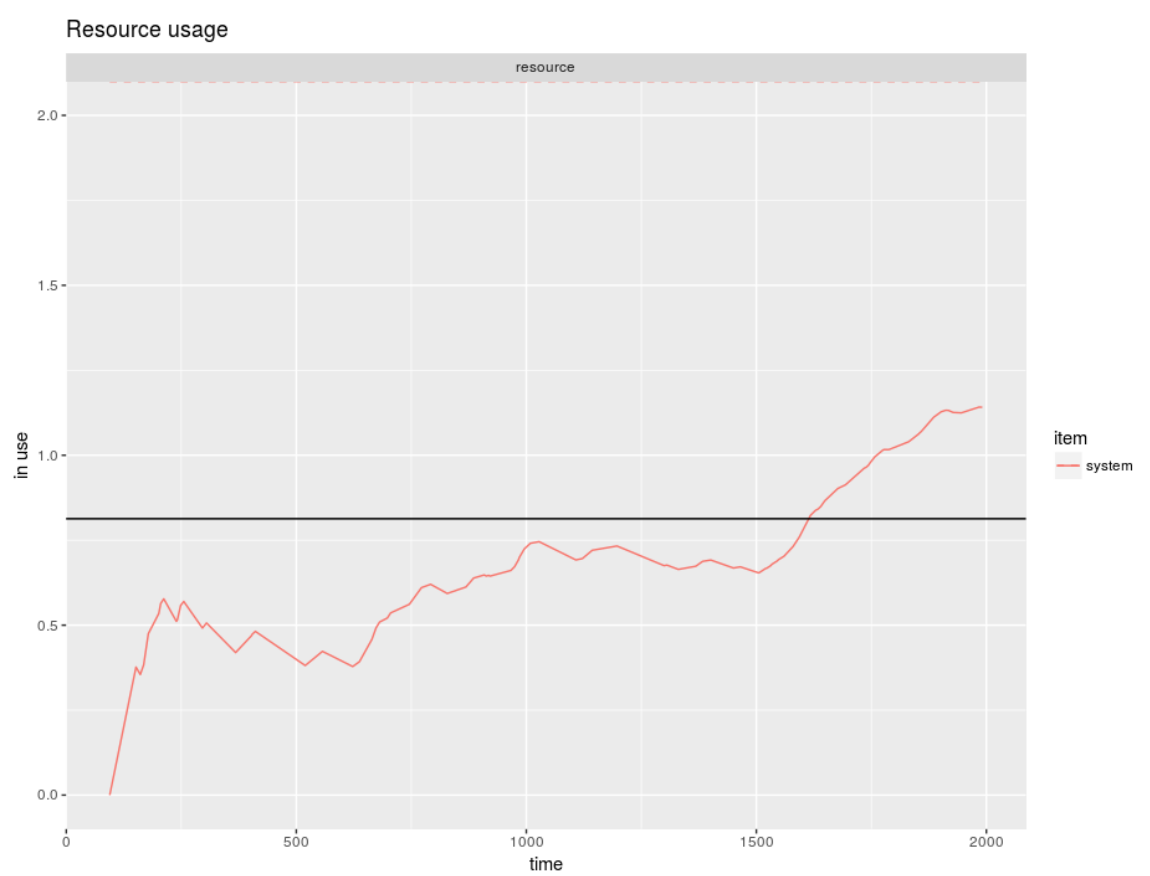

(e)

Figure 4. (a): Average Number of customers in the system with 1 ATM service point; (b): Average Number of customers in the system with 2 ATM service points; (c): Average Number of customers in the system with 3 ATM service points; (d): Average Number of customers in the system with 4 ATM service points and (e): Average Number of customers in the system with 5 ATM service points.

b. Average numbers of customers in the queue

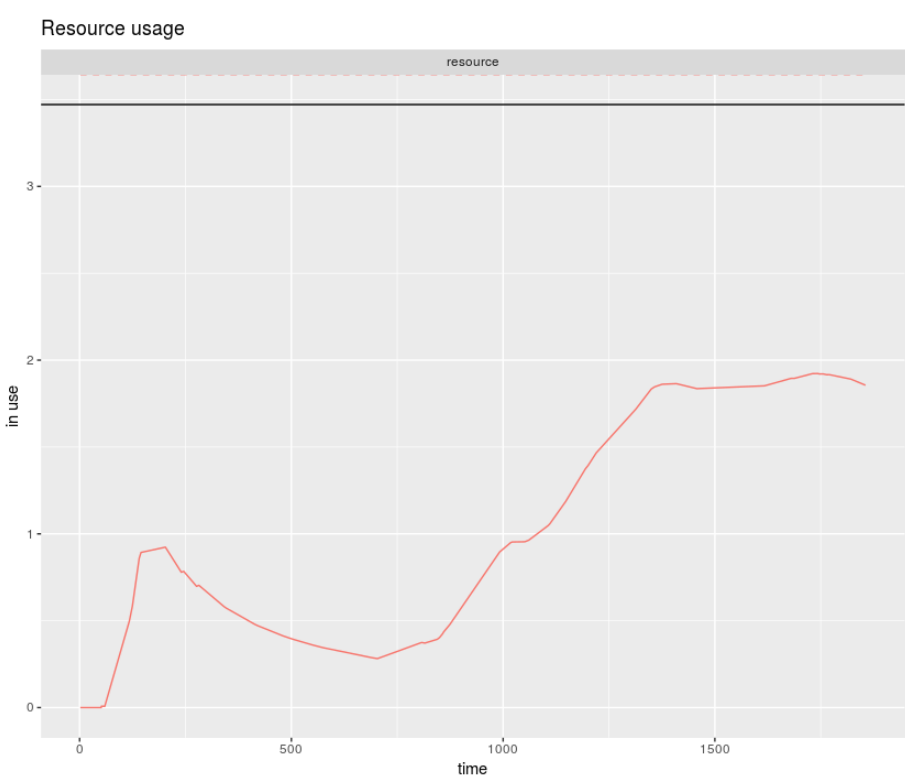

(a)

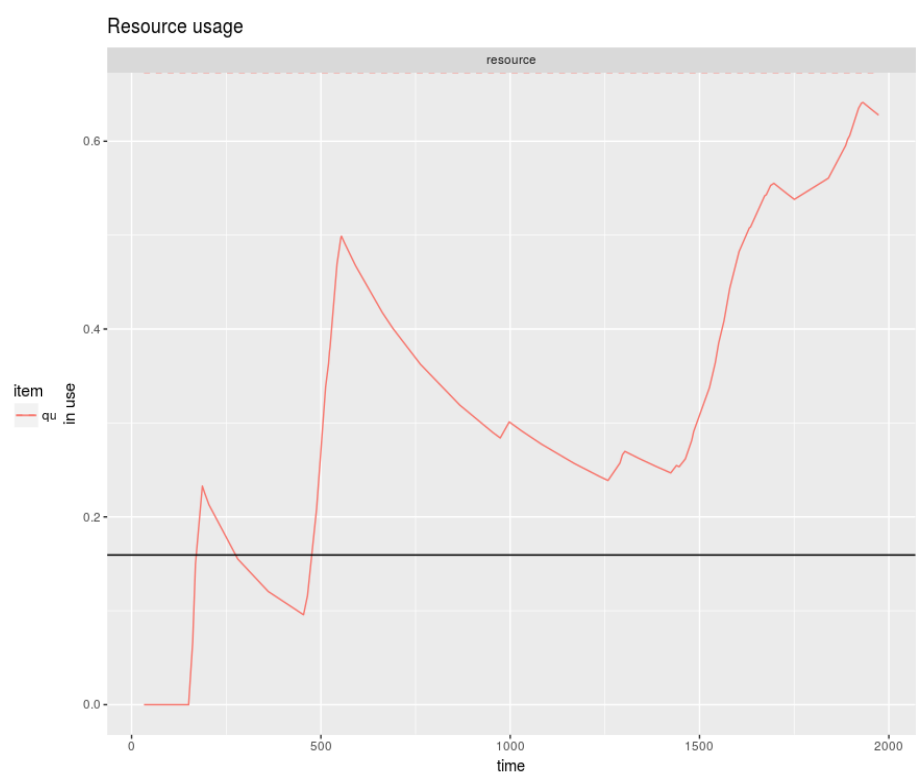

(b) 


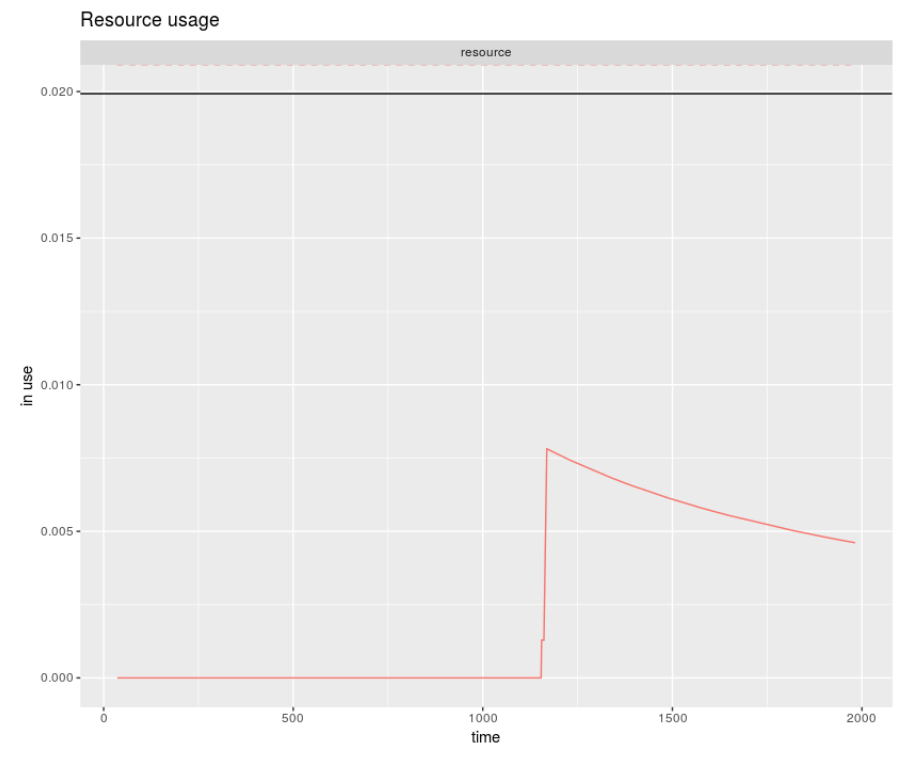

(c)

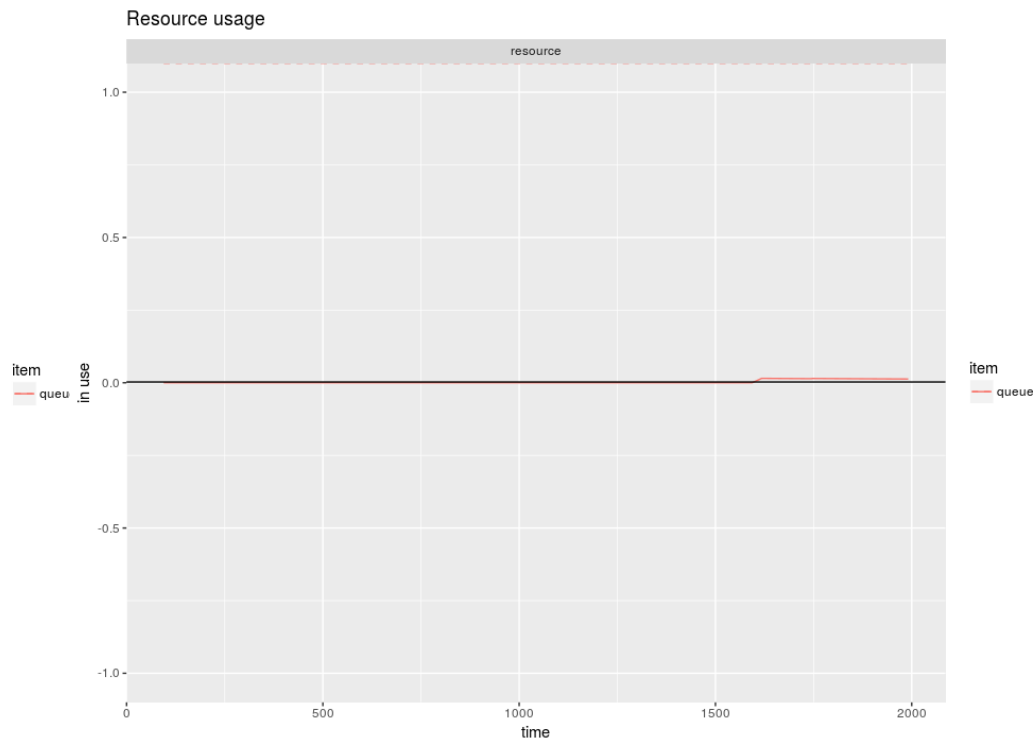

(d)

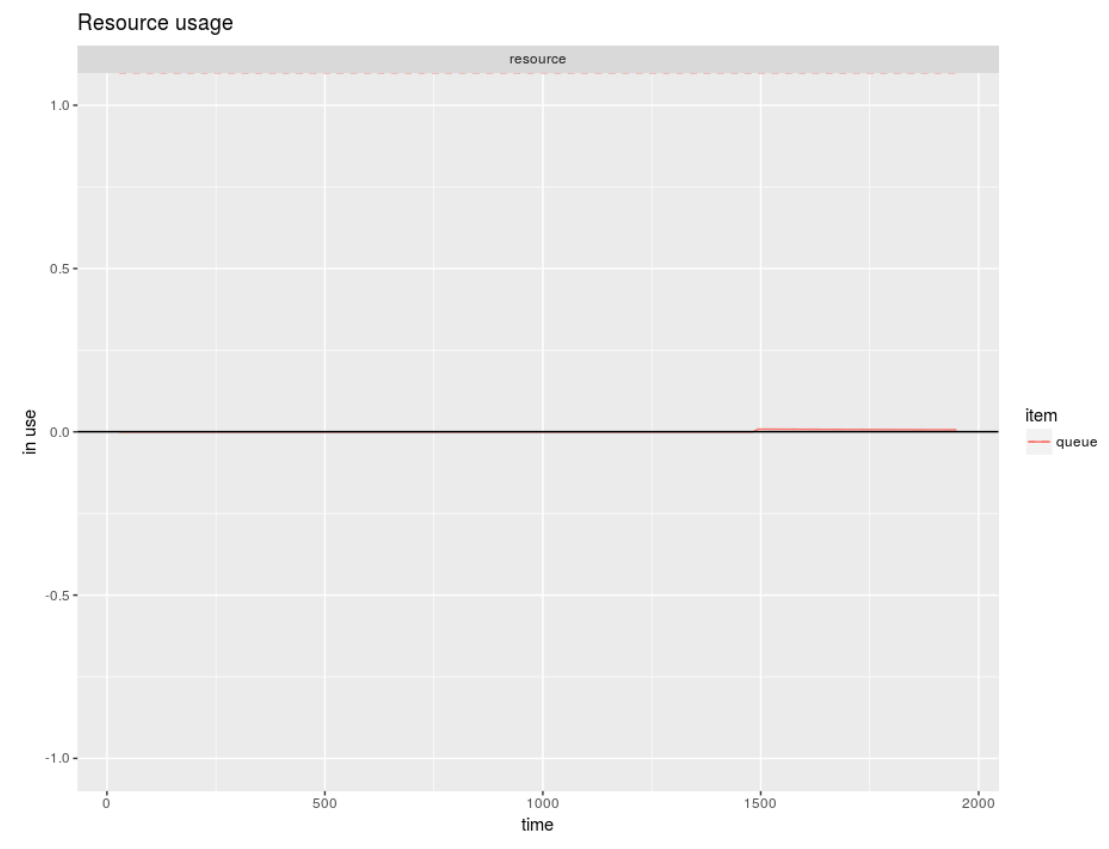

(e)

Figure 5. (a): Average Number of customers in the queue with 1 ATM service point; (b): Average Number of customers in the queue with 2 ATM service points; (c): Average Number of customers in the queue with 3 ATM service points; (d): Average Number of customers in the queue with 4 ATM service points and (e): Average Number of customers in the queue with 5 ATM service points. 


\section{c. Server Utilization in relation to Queue}

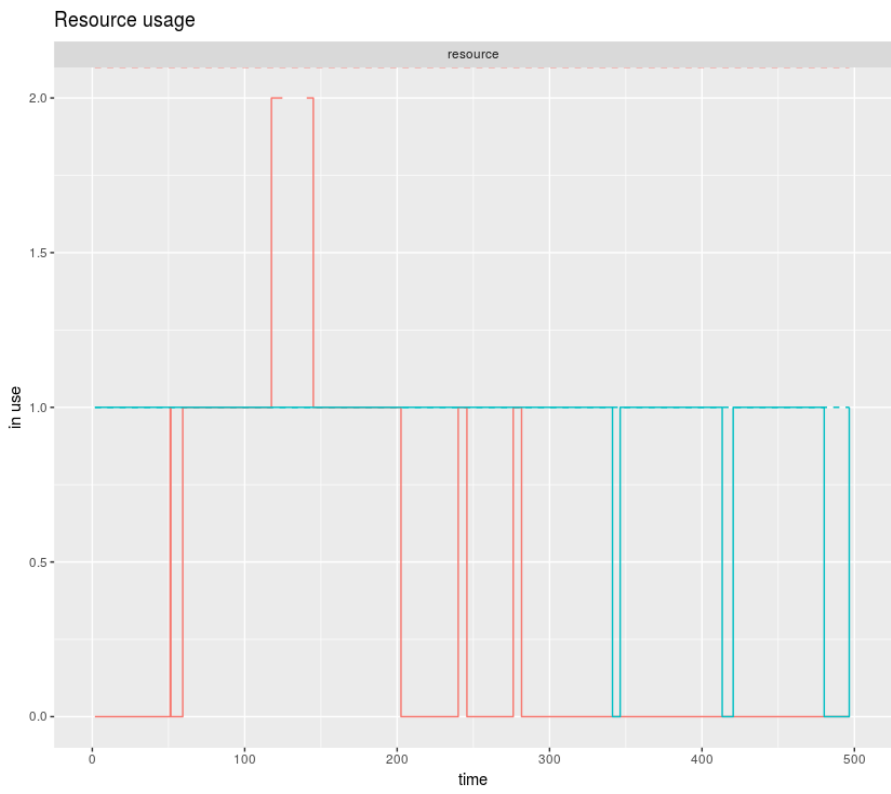

(a)

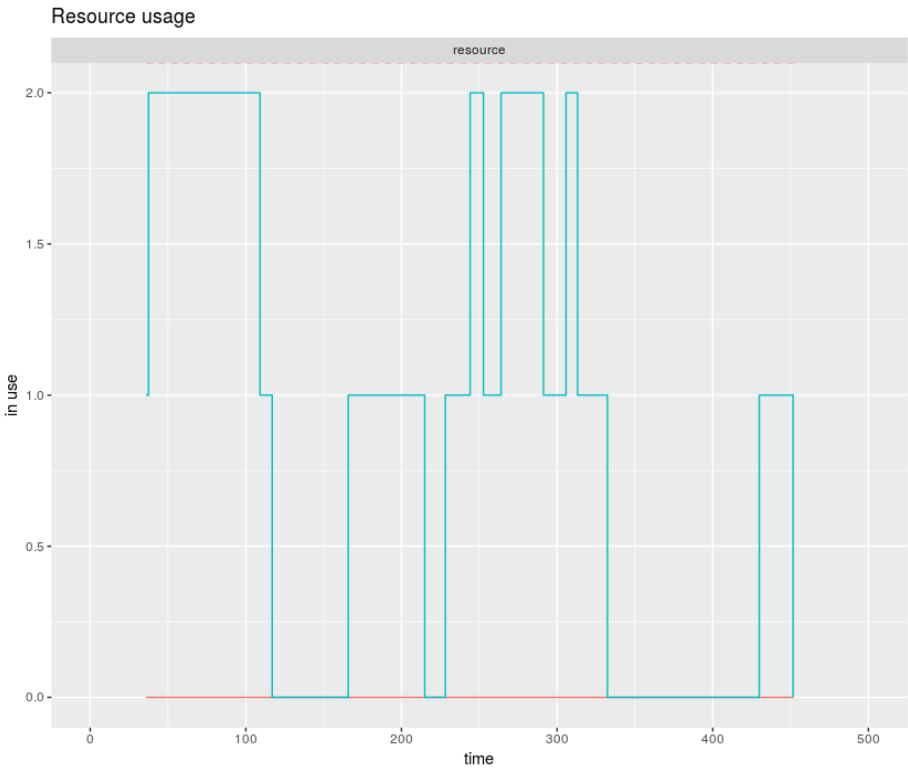

(c)

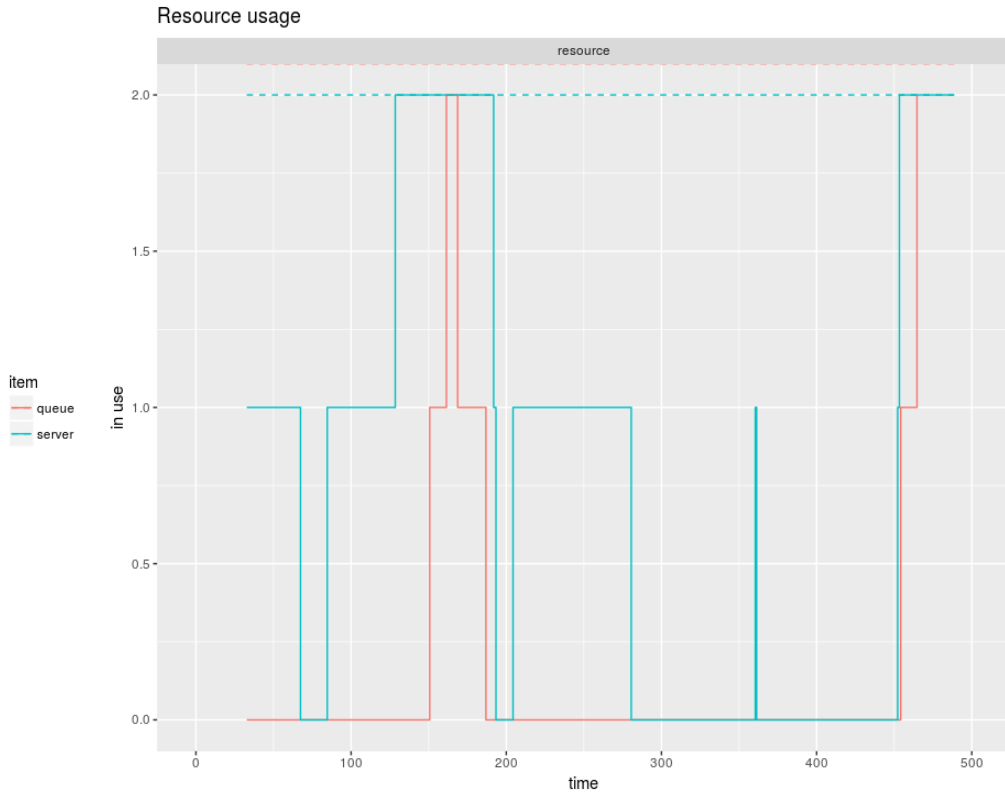

(b)

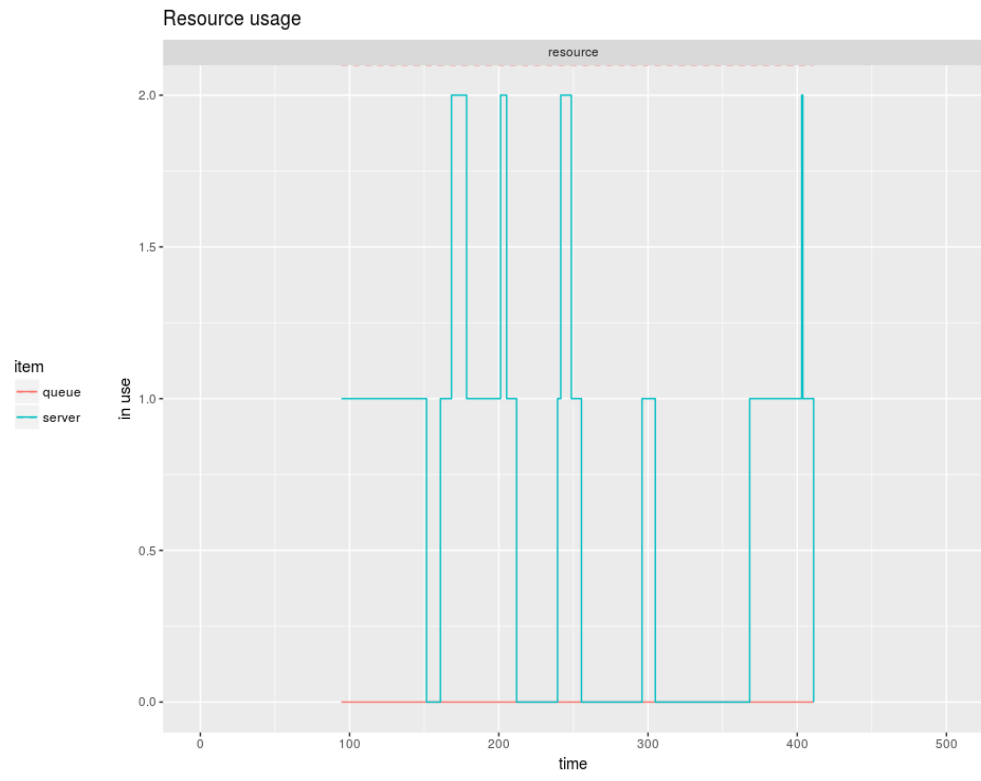

(d) 


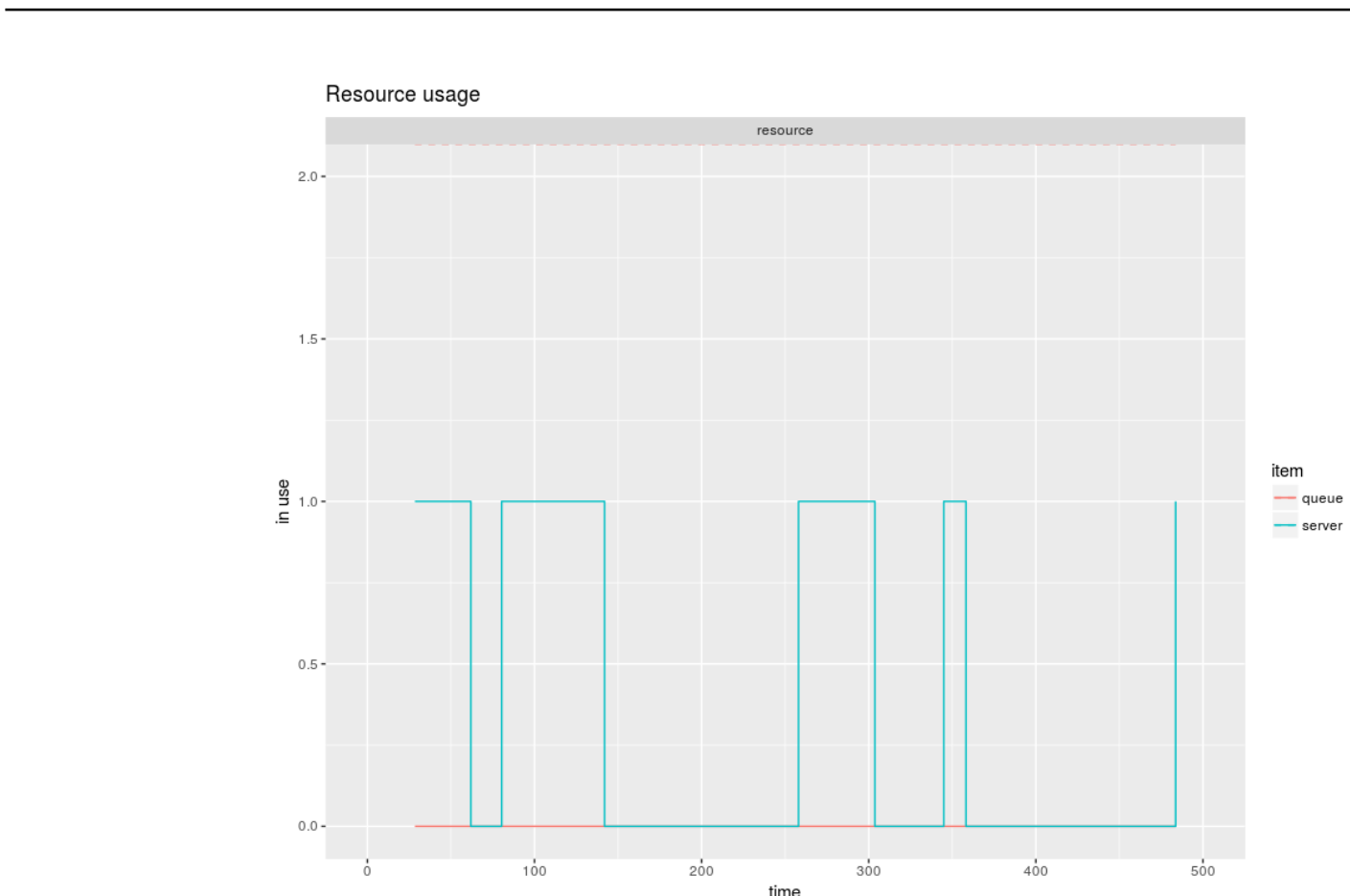

(e)

Figure 6. (a): Server utilization in relation to queue with 1 ATM service point; (b): Server utilization in relation to queue with 2 ATM service points; (c): Server utilization in relation to queue with 3 ATM service points; (d): Server utilization in relation to queue with 4 ATM service points and (e): Server utilization in relation to queue with 5 ATM service points.

In summary, the average number of customers in the system as provided in Figures 4 (a)-(e) are observed to be stable at the point when the server is 3 and above. Meanwhile a buildup of the queue will ultimately increase the service time. This was observed with 3 and 4 servers. Though this is not likely to happen with 5 servers with same time frame. Furthermore, with the experiment as depicted in Figures 5 (a)- (e), it is expected that the system (i.e. server points) will be able to cope with average customer with 4 servers. At this point the system is expected to handle queue flow with optimum service performance. Considering, the server utilization to queue build up and available service points, the experience as shown in figures 6 (a)-(e), server utilization is stochastically achieved as the number of servers are there and above. Less queue is probably experience with five servers.

\section{Conclusion}

Discrete-event simulation (DES) as proof to be applicable mechanism to models the operation of a system as a discrete sequence of events in time and occurrence. This approach is implemented in the paper to ascertain the validity of assertions provided in the work of Johnson et al (2018). With field data used, simulation experiments show an approximate trend of events similar to the stochastic method. There were serious identifiable challenges of system performance and server utilization with one server and two servers' deployment in ratio to the current population. Noticeable queue is also experimentally observed with three servers. From the experiment, a four, five servers' deployment stands as the check point to lessen the problem of congestion and other related vices. Hence a realistic result and model generated by the work of Johnson et al (2018) could be adopted for planning future deployment of ATM point in densely populated area such as the campus under study. 


\section{References}

Babulak. E (2008) "Trends in Discrete Event Simulations," 2008 Second UKSIM European Symposium on Computer Modeling and Simulation, Liverpool, 2008, pp. 1-1. doi: 10.1109/EMS.2008.107

Bakari H.R, Chamalwa H, A and Baba A.M (2014), “Queueing Process and Its Application to Customer Service Delivery: A case Study of Fidelity Bank Plc, Maiduguri", International Journal of Mathematics and Statistics Invention, Volume 2 Issue 1, pp 14-21.

Brian J. Huffman (2014) “An Object-Oriented Version of SIMLIB (a Simple Simulation Package)", School of Business University of Wisconsin - River Falls, retrieved 31st , Oct 2014 http://archive.ite.journal.informs.org/Vol2No1/Huffman/Huffman.php

Discrete Event Simulation (DES): https://en.wikipedia.org/wiki/DES

Hira D.S and Gupta P.K. (2004), "Simulation and Queueing Theory Operation Research S.C Chand and Company Ltd, New Delhi, India

Hiray J. (2008), “Waiting Lines and Queueing System”, Article of Business Management.

Inestopedia (2014), “Queueing Definition”, retrieved 26th $\quad$ Oct, $2014 \quad$ from http://www.investopedia.com/terms/q/queueing-theory.asp.

Johnson O. V., Aladesote O.I and Aliu H. (2018), Modeling a Life Queue-Event Data for Selected ATM ServicePoint(s), Journal of Mathematical Theory and Modeling, The International Institute for Science, Technology and Education (IISTE). ISSN 2224-5804 (Paper) ISSN 2225-0522 (Online) Vol.8, No.2, 2018.

Prateek S (2015), “Discrete-Event Simulation”, International Journal of Scientific \& Technology Research Volume 4, Issue 04, April 2015 ISSN 2277-8616.

Taha A.H (2003), “Operation Research: An Introduction, 7th Ed., Prentice Hall, India.

Vasumathi A. and Dhanavanthan P. (2010), “Application of Simulation Technique in Queueing Model for ATM Facility”, International Journal of Applied Engineering Research, Dindigul, Volume 1 No 30, pp 469-482.

Shannon, R.E., 1975. Systems Simulation - The Art and Science, Prentice-Hall.

Ucar, Smeets and Azcorra (2017), simmer: Discrete-Event Simulation for R, arXiv:1705.09746v2 [stat.CO] 5 Dec 2017. 


\section{Appendix I}

Table 1.0: The summarized queue metrics

\begin{tabular}{|c|c|c|c|c|c|c|c|c|}
\hline days & $n S$ & $p$ & $L$ & $L q$ & $W q$ & $w$ & Wiq & Lqq \\
\hline 1 & 1 & 0.9 & 8.97 & 8.07 & 378.96 & 421.21 & 421.21 & 9.97 \\
\hline 1 & 2 & 0.45 & 1.13 & 0.228 & 10.72 & 52.97 & 38.4 & 1.82 \\
\hline 1 & 3 & 0.3 & 0.93 & 0.03 & 1.4 & 43.66 & 20.12 & 1.43 \\
\hline 1 & 4 & 0.23 & 0.9 & 0.004 & 0.2 & 42.44 & 13.63 & 1.29 \\
\hline 1 & 5 & 0.18 & 0.9 & 0.001 & 0.03 & 42.27 & 10.3 & 1.22 \\
\hline$* 2$ & 1 & 1.06 & $\mathrm{n} / \mathrm{a}$ & $\mathrm{n} / \mathrm{a}$ & $\mathrm{n} / \mathrm{a}$ & $\mathrm{n} / \mathrm{a}$ & $\mathrm{n} / \mathrm{a}$ & $\mathrm{n} / \mathrm{a}$ \\
\hline 2 & 2 & 0.53 & 1.46 & 0.407 & 23.5 & 84.46 & 64.51 & 2.11 \\
\hline 2 & 3 & 0.35 & 1.11 & 0.056 & 3.25 & 64.21 & 31.34 & 1.54 \\
\hline 2 & 4 & 0.26 & 1.06 & 0.01 & 0.5 & 61.47 & 20.7 & 1.35 \\
\hline 2 & 5 & 0.21 & 1.06 & 0.001 & 0.07 & 61.04 & 15.45 & 1.27 \\
\hline 3 & 1 & 0.8 & 3.98 & 3.18 & 159.84 & 200 & 200 & 4.98 \\
\hline 3 & 2 & 0.4 & 0.95 & 0.152 & 7.63 & 47.8 & 33.45 & 1.67 \\
\hline 3 & 3 & 0.27 & 0.82 & 0.019 & 0.95 & 41.11 & 18.25 & 1.36 \\
\hline 3 & 4 & 0.2 & 0.8 & 0.002 & 0.12 & 40.28 & 12.55 & 1.25 \\
\hline 3 & 5 & 0.16 & 0.79 & 0 & 0.01 & 40.18 & 9.56 & 1.19 \\
\hline$* 4$ & 1 & 1.68 & $\mathrm{n} / \mathrm{a}$ & $\mathrm{n} / \mathrm{a}$ & $\mathrm{n} / \mathrm{a}$ & $\mathrm{n} / \mathrm{a}$ & $\mathrm{n} / \mathrm{a}$ & $\mathrm{n} / \mathrm{a}$ \\
\hline 4 & 2 & 0.83 & 5.67 & 3.987 & 169.09 & 240.24 & 220.9 & 6.21 \\
\hline 4 & 3 & 0.56 & 2.06 & 0.386 & 16.37 & 87.53 & 53.82 & 2.27 \\
\hline 4 & 4 & 0.42 & 1.75 & 0.076 & 3.2 & 74.36 & 30.64 & 1.72 \\
\hline 4 & 5 & 0.34 & 1.69 & 0.016 & 0.66 & 71.82 & 21.42 & 1.51 \\
\hline 5 & 1 & 0.65 & 1.84 & 1.188 & 87.35 & 134.95 & 134.95 & 2.83 \\
\hline 5 & 2 & 0.32 & 0.72 & 0.076 & 5.57 & 53.17 & 35.19 & 1.48 \\
\hline 5 & 3 & 0.22 & 0.66 & 0.008 & 0.61 & 48.21 & 20.23 & 1.28 \\
\hline 5 & 4 & 0.16 & 0.65 & 0.001 & 0.06 & 47.67 & 14.2 & 1.19 \\
\hline 5 & 5 & 0.13 & 0.65 & 0 & 0.01 & 47.61 & 10.93 & 1.49 \\
\hline 6 & 1 & 0.75 & 2.99 & 2.241 & 142.03 & 189.52 & 189.52 & 3.99 \\
\hline
\end{tabular}




\begin{tabular}{|c|c|c|c|c|c|c|c|c|}
\hline 6 & 2 & 0.38 & 0.87 & 0.122 & 7.76 & 55.25 & 37.98 & 1.6 \\
\hline 6 & 3 & 0.25 & 0.76 & 0.015 & 0.93 & 48.42 & 21.1 & 1.33 \\
\hline 6 & 4 & 0.19 & 0.75 & 0.002 & 0.11 & 47.61 & 14.61 & 1.23 \\
\hline 6 & 5 & 0.15 & 0.75 & 0 & 0.01 & 47.51 & 11.17 & 1.18 \\
\hline 7 & 1 & 0.74 & 2.86 & 2.116 & 122.28 & 165.08 & 165.08 & 3.86 \\
\hline 7 & 2 & 0.37 & 0.86 & 0.118 & 6.8 & 49.6 & 33.99 & 1.59 \\
\hline 7 & 3 & 0.25 & 0.76 & 0.014 & 0.81 & 43.61 & 18.94 & 1.33 \\
\hline 7 & 4 & 0.19 & 0.74 & 0.002 & 0.1 & 42.89 & 13.13 & 1.23 \\
\hline 7 & 5 & 0.15 & 0.74 & 0 & 0.01 & 42.81 & 10.05 & 1.17 \\
\hline 8 & 1 & 0.87 & 6.49 & 5.62 & 252.9 & 291.9 & 291.9 & 7.49 \\
\hline 8 & 2 & 0.43 & 1.07 & 0.2 & 9.01 & 48 & 34.4 & 1.76 \\
\hline 8 & 3 & 0.29 & 0.89 & 0.026 & 1.16 & 40.15 & 18.27 & 1.41 \\
\hline 8 & 4 & 0.22 & 0.87 & 0.003 & 0.16 & 39.15 & 12.44 & 1.28 \\
\hline 8 & 5 & 0.17 & 0.87 & 0 & 0.02 & 39 & 9.43 & 1.21 \\
\hline 9 & 1 & 0.78 & 3.42 & 2.643 & 205.21 & 265.28 & 265.28 & 4.42 \\
\hline 9 & 2 & 0.39 & 0.91 & 0.137 & 10.57 & 70.63 & 48.97 & 1.63 \\
\hline 9 & 3 & 0.26 & 0.79 & 0.017 & 1.29 & 61.35 & 26.98 & 1.35 \\
\hline 9 & 4 & 0.19 & 0.78 & 0.002 & 0.16 & 60.22 & 18.62 & 1.24 \\
\hline 9 & 5 & 0.16 & 0.78 & 0 & 0.02 & 60.08 & 14.21 & 1.18 \\
\hline 10 & 1 & 0.73 & 2.72 & 1.989 & 126.48 & 172.97 & 172.97 & 3.72 \\
\hline 10 & 2 & 0.37 & 0.84 & 0.112 & 7.17 & 53.66 & 36.64 & 1.58 \\
\hline 10 & 3 & 0.24 & 0.75 & 0.013 & 0.85 & 47.34 & 20.49 & 1.32 \\
\hline 10 & 4 & 0.18 & 0.73 & 0.002 & 0.1 & 46.59 & 14.22 & 1.22 \\
\hline 10 & 5 & 0.15 & 0.73 & 0 & 0.01 & 46.5 & 10.89 & 1.17 \\
\hline 11 & 1 & 0.53 & 1.13 & 0.601 & 54.54 & 102.73 & 102.73 & 2.13 \\
\hline 11 & 2 & 0.27 & 0.57 & 0.04 & 3.65 & 51.84 & 32.8 & 1.36 \\
\hline 11 & 3 & 0.18 & 0.54 & 0.004 & 0.35 & 48.54 & 19.52 & 1.21 \\
\hline 11 & 4 & 0.13 & 0.53 & 0 & 0.03 & 48.22 & 13.89 & 1.15 \\
\hline 11 & 5 & 0.11 & 0.53 & 0 & 0 & 48.19 & 10.78 & 1.11 \\
\hline
\end{tabular}




\begin{tabular}{|c|c|c|c|c|c|c|c|c|}
\hline 12 & 1 & 0.81 & 4.24 & 3.432 & 205.09 & 253.45 & 253.4 & 5.24 \\
\hline 12 & 2 & 0.41 & 0.97 & 0.158 & 9.47 & 57.82 & 40.61 & 1.68 \\
\hline 12 & 3 & 0.27 & 0.83 & 0.02 & 1.18 & 49.54 & 22.07 & 1.37 \\
\hline 12 & 4 & 0.2 & 0.81 & 0.003 & 0.15 & 48.51 & 15.16 & 1.25 \\
\hline 12 & 5 & 0.16 & 0.81 & 0 & 0.02 & 48.37 & 11.54 & 1.19 \\
\hline 13 & 1 & 0.58 & 1.4 & 0.815 & 64.61 & 110.84 & 110.84 & 2.4 \\
\hline 13 & 2 & 0.29 & 0.64 & 0.054 & 4.29 & 50.52 & 32.62 & 1.41 \\
\hline 13 & 3 & 0.19 & 0.59 & 0.006 & 0.44 & 46.67 & 19.13 & 1.24 \\
\hline 13 & 4 & 0.15 & 0.58 & 0.001 & 0.04 & 46.27 & 13.53 & 1.17 \\
\hline 13 & 5 & 0.12 & 0.58 & 0 & 0 & 46.24 & 10.47 & 1.13 \\
\hline
\end{tabular}

\section{R Code}

library(simmer)

library(simmer.plot)

library(dplyr)

set.seed (1234)

\#Scripts to load all the csv files of the queue data from day 1 to 13

path <- "/home/ilanre/Documents/Queue Research Work/"

files <- list.files(path=path, pattern="*.csv")

for(file in files)

I

perpos <- which(strsplit(file, "'")[[1]]==".")

assign(

gsub(" ","'", substr(file, 1, perpos-1)),

read.csv(paste $($ path,file, sep $=" " \prime)))$

\}

lambda $=($ mean $($ Dayl $[, 2])+$

mean $(\operatorname{Day} 2[, 2])+$ mean $(\operatorname{Day} 3[, 2])+$ mean $(\operatorname{Day} 4[, 2])+$ mean $($ Day5 [,2] $)+$ mean $($ Day6[,2] $)+$ mean $($ Day $7[, 2])+$ mean $(D$ ay8[,2])+

mean $(\operatorname{Day} 9[, 2])+$ mean $(\operatorname{Day} 10[, 2])+$ mean $(\operatorname{Day} 11[, 2])+$ mean $(\operatorname{Day} 12[, 2])+$ mean $(\operatorname{Day} 13[, 2])) / 13$

$m u=(\operatorname{mean}(\operatorname{Day} 1[, 5])+$

mean $(\operatorname{Day} 2[, 5])+$ mean $(\operatorname{Day} 3[, 5])+$ mean $(\operatorname{Day} 4[, 5])+$ mean $(\operatorname{Day} 5[, 5])+$ mean $($ Day6[,5])+mean $($ Day $7[, 5])+$ mean $(D$ ay8[,5])+

mean $($ Day9[,5])+mean $($ Day10[,5])+mean $($ Dayl1 [,5] $)+$ mean $($ Day12[,5])+mean $($ Day13[,5]))/13

rho <- lambda/mu

$c=5$

mmc.trajectory <- trajectory() \%>\%

seize("resource", amount=1) \%>\%

timeout(function() $\operatorname{rexp}(1, m u)) \%>\%$

release("resource", amount=1)

mmc.env <- simmer() \%>\%

add_resource("resource", capacity $=c$, queue_size $=\operatorname{Inf}) \%>\%$

add_generator("arrival", mmc.trajectory, function() rexp(1, lambda)) \%>\% 


$$
\operatorname{run}(\text { until=2000) }
$$

\# Theoretical values

ind <- $0:(c-1)$

pi_o $<-1 /(\operatorname{sum}(r h o * *$ ind $/$ factorial $($ ind $))+($ rho $* *$ c/factorial $(c)) *(1 /(1-$ rho/c $)))$

$m m c . L<-r h o+\left((\right.$ rho $\left.* * c / f a c t o r i a l(c)) * p i \_0\right) *((r h o / c) /((1-r h o / c) * * 2))$

mmc. $L q<-m m c . L-r h o$

\# Evolution of the average number of customers in the system

plot(mmc.env, "resources", "usage", "resource", items="system") +

geom_hline $($ yintercept $=m m c . L)+y \lim (0,2)$

\# Evolution of the average number of customers in the queue

plot(mmc.env, "resources", "usage", "resource", items="queue") +

geom_hline $($ yintercept $=m m c . L q)+y \lim (-1,1)$

\# Visualization of the individual elements

plot(mmc.env, "resources", "usage", "resource", items=c("queue", "server"), steps=TRUE) + $x \lim (0,500)+y \lim (0,2)$

mmc.arrivals <-get_mon_arrivals(mmc.env)

mmc.t_system <-mmc.arrivals\$end_time - mmc.arrivals\$start_time

lambda_eff<-lambda

mmc. $W<-m m c . L /$ lambda_eff

mmc. $W ;$ mean(mmc.t_system)

\# Confidence Interval

envs <- mclapply(1:50, function $(i)\{$

simmer() $\%>\%$

add_resource("resource", capacity=c, queue_size=Inf) $\%>\%$

add_generator("arrival", mmc.trajectory, function() $\operatorname{rexp}(1, \operatorname{lambda})) \%>\%$

$\operatorname{run}(500) \%>\%$

wrap()

\}, mc.set.seed $=$ TRUE)

t_system $<-$ get_mon_arrivals(envs) $\%>\%$

dplyr::mutate $\left(t \_s y s t e m=e n d \_t i m e-s t a r t\right.$ time $) \%>\%$

dplyr::group_by(replication) $\%>\%$

dplyr::summarise $\left(\right.$ mean $=$ mean $\left(t_{-}\right.$system $\left.)\right)$

n_system <-get_mon_resources(envs)\%>\%

dplyr::group_by(replication) $\%>\%$

dplyr::summarise $($ mean $=\operatorname{sum}($ head $($ system,-1$) * \operatorname{diff}($ time $)) / \operatorname{tail}($ time, 1$))$

t.test $\left(t \_s y s t e m \$\right.$ mean)

t.test $\left(n \_s y s t e m \$ m e a n\right)$

lambda; 1/mean(diff(subset(mmc.arrivals, finished==TRUE)\$start_time )) 